\title{
Raumaneignungen, Regeln und Profite in Dhakas Feld des Straßenhandels - Sozialgeographische Erklärungsversuche auf Grundlage von Bourdieus Theorie der Praxis
}

\author{
B. Etzold \\ Geographisches Institut der Universität Bonn, Meckenheimer Allee 166, 53115 Bonn, Germany \\ Correspondence to: B. Etzold (etzold@giub.uni-bonn.de)
}

Received: 3 November 2012 - Revised: 20 August 2013 - Accepted: 21 August 2013 - Published: 3 April 2014

\begin{abstract}
Kurzfassung. The paper discusses street vendors' spatial appropriations and the governance of public space in Dhaka, the capital of Bangladesh. The much debated question in social geography how people's position in social space relates to their position in physical space (and vice versa) stands at the centre of the analysis. I use Bourdieu's Theory of Practice to discuss this dialectic relation at two analytical levels. On a micro-political level it is shown that the street vendors' social positions and the informal rules of the street structure their access to public space and thus determine their "spatial profits". At a macro-political level, it is not only the conditions inside the "field of street vending" that matter for the hawkers, but also their relation to the statecontrolled "field of power". The paper demonstrates that Bourdieu's key ideas can be linked to current debates about spatial appropriation and informality. Moreover, I argue that Bourdieu's theory builds an appropriate basis for a relational, critical, and reflexive social geography in the Urban South.
\end{abstract}

Im Beitrag geht es um die Raumaneignungen von StraBenhändlern und die Regulation öffentlicher Räume in Dhaka, der Hauptstadt von Bangladesch. Im Zentrum der Analyse steht die in der Sozialgeographie durchaus umstrittene These, dass sich die gesellschaftliche Verteilung von Macht in den physischen räumlichen Strukturen widerspiegelt, und umgekehrt. Die wechselseitige Beziehung zwischen der Position von Akteuren im sozialen und im physischen Raum wird auf der Grundlage von Bourdieus Theorie der Praxis auf zwei analytischen Ebenen verdeutlicht. Aus einer mikropolitischen Perspektive zeigt sich, dass die sozialen Positionen der Händler sowie informelle Regeln festlegen wer sich den öffentlichen Raum aneignen und somit ,Raumprofite“ erzielen kann. Auf einer makro-politischen Ebene wird veranschaulicht, dass nicht nur die Bedingungen innerhalb des „Feldes des Straßenhandels“ bedeutsam sind, sondern auch die Relationen der subalternen Akteure zum staatlich kontrollierten „Feld der Macht“. Bourdieus Theorie ist anschlussfähig zu aktuellen Diskussionen um Raumaneignung und Informalität. Darüber hinaus bietet sie eine konzeptio- nelle Grundlage für eine relationale, kritische und reflexive Sozialgeographie im urbanen Süden.

\section{Einleitung}

Konflikte um die Planung, Nutzung und Regulation städtischer Räume sind ein immanenter Bestandteil der Urbanisierung und stellen Stadtplaner wie Politiker und nicht zuletzt die Stadtbewohner selbst vor größte Herausforderungen. Dies zeigt sich im besonderen Maße in öffentlichen Räumen, in deren Ausgestaltung und Nutzung sich Machtverhältnisse und Teilhabemöglichkeiten sowie Spannungen und Bruchlinien in der städtischen Gesellschaft widerspiegeln - egal ob es sich dabei um Metropolen des Nordens (vgl. Madanipour, 2003; Low und Smith, 2006) oder des Süden (vgl. Brown, 2006; Madanipour, 2010) handelt. Der Straßenhandel, und insbesondere der Verkauf von zubereitetem Essen im öffentlichen Raum, gehört in vielen Metropolen des Südens, wie Mumbai (Anjaria, 2010), Hyderabad (Dittrich, 2008), Caracas (Garcia-Rincon, 2007) oder Recife (Redepenning et al., 
2010), zum Alltag und Stadtbild. Dennoch entzünden sich gerade an diesem Thema hitzige Debatten um Raumaneignungen und die ,angemessene“ Form der städtischen Regulation. Straßenverkäufer sind einerseits auf den Zugang zum öffentlichen Raum angewiesen, um ihr Leben zu sichern und die Stadt mit Gütern zu versorgen (vgl. Brown, 2006; Bhowmik, 2010). Andererseits passen die informellen Raumaneignungen der Straßenhändler, und im weiteren Sinne der Lebensalltag der ärmeren Bevölkerung, nicht zu den Zukunftsvisionen der Eliten von modernen, schönen und sauberen Städten. Entwicklungspläne und „Verschönerungsaktionen“ gehen daher oftmals mit der Bekämpfung von subalternen Raumaneignungen einher - Slums werden geräumt, die StraBenhändler werden verdrängt (vgl. Cross und Karides, 2007; Davis, 2007; Bohle und Sakdapolrak, 2008).

Im Beitrag werden die alltäglichen Raumaneignungen von Straßenhändlern in Dhaka, die Regulation öffentlicher Räume und die „Raumprofite“ im „Feld des Straßenhandels“ mit Hilfe der Theorie der Praxis von Pierre Bourdieu erfasst. Im Zentrum der Analyse steht die in der Sozialgeographie durchaus umstrittene These, dass sich gesellschaftliche Ordnungen und Machtstrukturen in den physischen räumlichen Strukturen, d.h. auch in den Positionen von Akteuren im Raum, widerspiegeln. Im Fazit wird gefragt, ob Bourdieus Theorie eine geeignete Grundlage für eine relationale, kritische und reflexive Sozialgeographie im urbanen Süden bietet und ob sie anschlussfähig ist zu aktuellen Diskussionen um Raumaneignung und Informalität im Sinne von postkolonialen Konzepten des subaltern urbanism.

\section{2 Öffentliche Räume als Arenen der Raumaneignung}

\subsection{Umkämpfte öffentliche Räume}

Städtische öffentliche Räume sind die der Öffentlichkeit frei zugänglichen Orte einer Stadt. Verschiedene Typen können entsprechend ihrer Zugangs- und Nutzungsbedingungen auf einem Kontinuum von gänzlich öffentlichen Räumen, wie Plätzen oder Parks, zu gänzlich privaten Räumen, wie Einkaufszentren oder Cafés, eingeordnet werden (vgl. Frey, 2004; Klamt, 2012). Im Begriff des öffentlichen Raumes manifestieren sich aber auch Spannungen zwischen konkreten Orten und Praktiken und der diffusen „Unräumlichkeit“ der öffentlichen Meinung und politischer Diskurse (Low und Smith, 2006:3). Sind öffentliche Räume umkämpft, steht daher meistens nicht der Raum an sich im Zentrum des Konfliktes. Es geht erstens eher um die „Raumprofite“ (Bourdieu, 1991) die erzielt werden können, seien es die Renditen der Investoren der um einen attraktiven Platz gelegenen Gebäude, oder um die Gewinne eines Straßenhändlers, der auf die Nutzung des öffentlichen Raumes zur Lebenssicherung angewiesen ist (vgl. Brown, 2006). Zweitens geht es um Politik; die Macht, öffentliche Räume zu gestalten und zu kontrollieren. Diese Macht scheint sich momentan in vielen Städten von den Stadtverwaltungen beziehungsweise der Kom- munalpolitik hin zu privaten Unternehmen, Shopping MallBetreibern und Sicherheitsfirmen zu verschieben (vgl. Glasze et al., 2005; Low und Smith, 2006). Drittens geht es um Repräsentation, denn öffentliche Räume sind wichtige Bühnen auf denen um gesellschaftliche Anerkennung gekämpft wird (vgl. Mitchell, 2003). Letztlich lassen der Umfang, die Dauer und die Weise wie - kapitalintensiv und staatlich geschützt oder möglicherweise subversiv und illegal - sich Akteure Räume aneignen sowie die Intensität der Konflikte um eine „,angemessene Nutzungen öffentlicher Räume“ (Cross und Karides, 2007) Rückschlüsse über herrschende Machtverhältnisse in einer Stadt zu (Frey, 2004). Über öffentliche Räume und ihre Aneignung nachzudenken erfordert daher die „Ideale und Realitäten, Paradigmen und Paradoxien der Stadtgesellschaft zu analysieren, [...] und diese räumlich und gesellschaftlich zu erklären“ (Klamt, 2012:775). Bourdieus Theorie der Praxis bietet hierfür viele Anknüpfungspunkte.

\subsection{Raumaneignungen im urbanen Süden - Erklärungsversuche mit Bourdieu}

Bourdieus Theorie der Praxis (u.a. 1976, 1998) bietet ein in sich schlüssiges Gedankengebäude, um die Handlungen von Menschen in ihrer gesellschaftlichen Einbettung zu verstehen, um ,verborgene Mechanismen der Macht“ zu entschlüsseln, und um zu erfassen wie sich soziale Strukturen räumlich manifestieren. Dies sind letztlich auch die Kernanliegen der Sozialgeographie und der geographischen Entwicklungsforschung. Daher hat Bourdieus Theorie die konzeptionellen Diskussionen in beiden Subdisziplinen entscheidend beeinflusst (vgl. Werlen, 1997; Lippuner, 2005b; Werlen und Lippuner, 2011; bzw. Dörfler et al., 2003; Graefe und Hassler, 2006; Deffner und Haferburg, in dieser Ausgabe; Sakdapolrak, in dieser Ausgabe). Bourdieus relationale Raumkategorien (Bourdieu, 1991, 2005b; Lippuner, 2005a; Schroer, 2006) sind ein hilfreiches Instrumentarium zur Analyse der Aneignung öffentlicher Räume (vgl. Frey, 2004; Etzold, 2011). Die Materialität des Raumes kann ebenso berücksichtigt werden wie die Beziehungen der im Raum agierenden Akteure, die vorherrschenden Modi der Kontrolle, sowie die Diskurse um die symbolische Bedeutung von Räumen und ihrer bevorzugten Nutzung. Im physischen oder materiellen Raum bestimmen die Naturgesetze die absoluten Positionen der physischen Elemente zueinander (Bourdieu, 2005b). Der soziale Raum oder das Feld ist nur in seiner Relationalität denkbar, denn Akteure nehmen auf Grundlage ihrer Ausstattung mit ökonomischem, sozialem, kulturellem und symbolischem Kapital spezifische Positionen in „Kraftfeldern“ (Bourdieu, 1998:49) ein. Grundsätzlich ist Kapital - und dementsprechend auch Machtpotentiale - in Feldern ungleich verteilt. Akteure haben ihre relative Position im Feld verinnerlicht. Sie nehmen dementsprechend ihr (Um)Feld wahr, bewerten ihre Handlungsoptionen und agieren so mit einem spezifischen Habitus (Bourdieu und Wacquant, 1996). Mit dem so genannten angeeigneten physischen Raum 


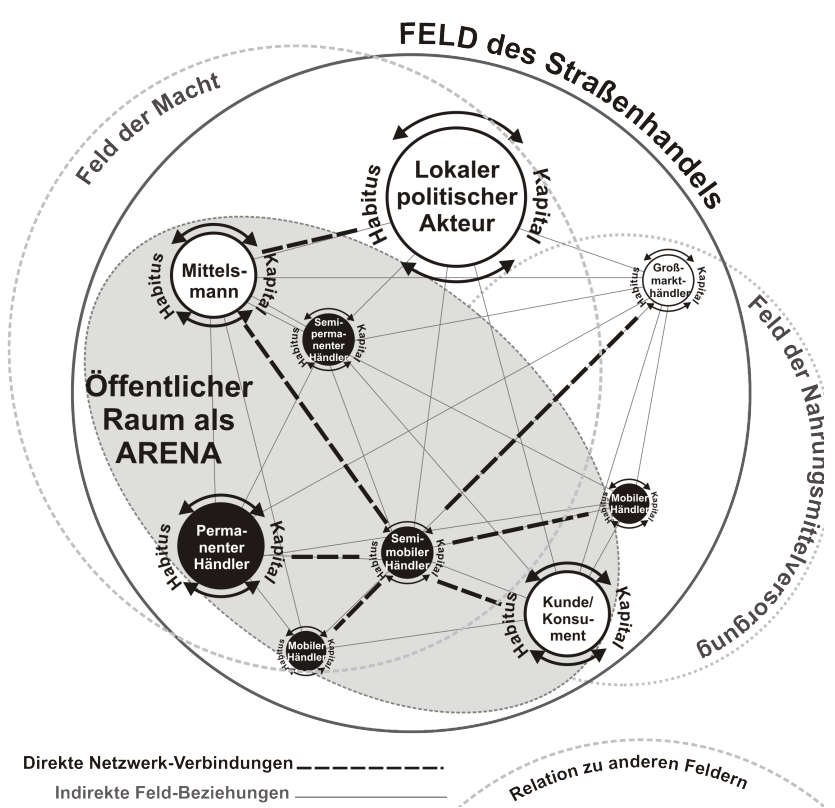

Abb. 1. Relative Positionen von Akteuren im Feld des Straßenhandles und in den Arenen des öffentlichen Raumes sowie Beziehungen zu anderen Feldern. Quelle: Eigene Darstellung angelehnt an Sakdapolrak (2010:61) und Etzold et al. (2009:6).

bezeichnet Bourdieu die realisierte Verteilung von Gütern und Dienstleistungen und die Verortung von Akteuren und Gruppen im physischen Raum. Entsprechend ihrer Position können sich Akteure Raum aneignen und ,räumliche Profite“ (Bourdieu, 2005b:120) erzielen - oder eben nicht. Dieser angeeignete physische Raum, den ich im Folgenden als Arena bezeichne (vgl. Etzold et al., 2009), ist zum einen das Ergebnis von sozialen Praktiken und zum anderen Gegenstand von Aushandlungsprozessen zwischen Akteuren mit unterschiedlichen Interessen und Machtpotentialen. Öffentliche Räume sind geeignete Beispiele einer Arena, denn hier werden soziale Positionen auf die Ebene des physischen Raumes projiziert (Abbildung 1).

Es ist common sense in der Sozialgeographie, dass sich das Soziale in räumlichen Strukturen „verfestigt“ und dass im Umkehrschluss viele soziale Prozesse nur in ihrer Räumlichkeit zu verstehen sind (vgl. u.a. Lippuner, 2005a; Werlen und Lippuner, 2011; Rothfuß, 2012). Gesellschaftliche Kräfteverhältnisse zeigen sich laut Bourdieu (2005b:118) insofern bei der Raumaneignung, als dass sich die „Position eines Akteurs im Sozialraum [...] in dem von ihm eingenommenen physischen Raum wider[spiegelt]“. Wenn Akteure in einer machtvollen Position in der Arena - sie sind mit der richtigen Kombination an Kapital ausgestattet - einen öffentlichen Raum besetzen und seine Nutzung kontrollieren, bedeutet das gleichzeitig auch, dass die Nutzung dieses Raumes anderen verwehrt bleibt. Nur sie können dann räumliche Profite erzielen und ,verräumlichen“ ihre soziale Stellung. Dies ist wiederum Ausdruck ihrer Macht, denn der ,mehr oder weniger ostentative Konsum von Raum ist ja eine Form der Zurschaustellung von Macht par excellence" (ebd.). Im Umkehrschluss könnte man daraus schließen, dass „der von einem Akteur eingenommene Ort und sein Platz im angeeigneten physischen Raum hervorragende Indikatoren für seine Stellung im sozialen Raum abgeben“ (Bourdieu, 1991:26). Einige Autoren kritisieren diese These als essentialistisch (u.a. Lippuner, 2005a:164; Dörfler, 2010:34), auch wenn Bourdieu explizit darlegt, dass die räumlichen Bedingungen den sozialen Raum nicht determinieren, sondern lediglich strukturieren (vgl. Schroer, 2006:89). Auch Lossau und Lippuner (2004:26) warnen vor dem „Treten in eine Raumfalle" und einer Gleichsetzung von räumlicher und sozialer Position. Vielmehr sollte mit empirischer Forschung dargelegt werden, ob die spezifische „Verortung“ von Akteuren im Raum wirklich Ausdruck ihrer sozialen Position ist, oder ob andere Faktoren die Raumaneignung beeinflussen. Genau dieser Zusammenhang zwischen der Position von Straßenhändlern in der Arena, im Sinne der realisierten Aneignung öffentlicher Räume, und ihrer Position im „Feld des Straßenhandels", im Sinne ihres Geschäftserfolgs und ihrer Anerkennung, steht im Zentrum dieses Beitrages.

\section{Methodik einer relationalen, kritischen und reflexiven Feld-Forschung}

Im Folgenden werden die methodologischen Konsequenzen der „Feld“-Forschung im Sinne der Theorie der Praxis aufgezeigt und mit der Darstellung der Methodik, der dieser Fallstudie zu Grunde liegenden Dissertation (Etzold, 2013), verknüpft. Eine relationale und kritische „Feld“-Forschung erfordert sechs analytische Schritte (erweitert nach Bourdieu und Wacquant, 1996). Erstens gilt es das Feld zu definieren, seine Grenzen abzustecken, und seine Geschichte zu rekonstruieren. Zur Analyse des „Feldes des Straßenhandels“ in Dhaka führte ich im Zeitraum von vier Jahren (2007 bis 2010) Interviews mit Akteuren, die den Straßenhandel in seinem Charakter prägen, d.h. in ihm „Feldeffekte“ erzeugen, wie Straßenhändler, Konsumenten oder Polizisten. Zweitens sollten die „Relationen zwischen den Positionen der in diesem Feld miteinander konkurrierenden Akteure" (ebd.) untersucht werden. Diese relativen Positionen wurden durch semi-strukturierte Interviews, Tiefengespräche und eine Umfrage von 120 Essensverkäufern an sechs Standorten erfasst. Drittens sollte man den Habitus von Akteuren analysieren, um zu verstehen, wie bestehende soziale und ökonomische Verhältnissen verinnerlicht wurden (ebd.: 136). In Interviews wurden die Lebenswege von Händlern rekonstruiert, um so ihre Handlungsdispositionen zu erfassen. Viertens gilt es die immanenten Regeln, ,die das normale Funktionieren des Feldes bestimmen, und damit auch die Profite, die sich in ihm erzielen lassen" (ebd.: 132), zu erkennen. Durch Interviews mit Händlern und lokalen politischen Akteuren konnten die Wirkungen von formalen Regeln und informellen „Gesetzen 
der Straße“ erfasst werden. Fünftens müssen die sozialen und räumlichen Praktiken analysiert werden. Beobachtungen, Kartierungen sowie Interviews ermöglichten es, Raumaneignungen der Händler und ihre Bewältigungsstrategien im Kontext von staatlicher Gewalt zu verstehen. Sechstens sollte das betrachtete Feld auch in Beziehung zu anderen sozialen Feldern gesetzt werden. Man solle insbesondere ,die Position des Feldes im Verhältnis zum Feld der Macht analysieren“ (ebd.: 136). Das „Feld der Macht“ ist der „Spielraum, in dem die Kapitalbesitzer vor allem um die Macht über den Staat kämpfen“ (Bourdieu, 1998:101). Die Beziehung eines Feldes zum von staatlichen Akteuren kontrollierten „Feld der Macht" ist nicht nur entscheidend für seine relative Bedeutung, sondern auch für die Logik seiner Regulation. Die aktuelle Gesetzeslage und Diskurse über den Straßenhandel wurden durch Gespräche mit Polizisten, Verwaltungsangestellten, Experten und durch Zeitungsartikel erfasst. Solch ein Zugang ist insofern als eine „Kritische Geographie der Machtbeziehungen“ (Kühne, 2008) zu sehen, als dass durch die Betrachtung der Gesetzgebung, politischer Ränkespiele und Diskurse wesentliche Aspekte der gesellschaftlichen und räumlichen Verteilung von Macht entschlüsselt werden können (vgl. u.a. Belina und Michel, 2008; Rothfuß, 2012).

Mit Bourdieu im globalen Süden zu forschen heißt allerdings auch, die eigene Position als Forscher selbstkritisch zu hinterfragen. So spiegeln sich asymmetrische postkoloniale Machtverhältnisse geradezu idealtypisch in einer Befragungssituation wider, in der ein weißer männlicher Akademiker aus Deutschland und ein bangladeschischer Student, der zugleich Übersetzer und „kultureller Mediator“ (Rothfuß, 2009:178) ist, einer Händlerin in einer Slum-Hütte gegenübersitzen. Bei der Feld-Forschung sollte man sich daher bewusst machen, dass ,,Sozialwissenschaft gar nicht neutral, indifferent und unpolitisch sein [kann]“" (Wacquant, 1996:82). Als Forschender tritt man in eine soziale Beziehung mit den Befragten ein und verändert so auch die Ergebnisse (Bourdieu, 2005a). Durch die eigenen Feldeffekte läuft man ständig Gefahr „Fehlinterpretationen über die handelnden Anderen zu produzieren“ (Rothfuß, 2009:173). Reflexivität bedeutet eine Bewusstwerdung der Relationen zwischen Beobachter und beobachteten Subjekten, eine Überprüfung der verwendeten Begriffe und Kategorien und somit eine selbstkritische Evaluation der ,wissenschaftlichen Beobachtungs- und Beschreibungspraxis“ (Lippuner, 2005b:142). Reflexivität ist somit kein Selbstzweck, sondern eine Grundbedingung sozialgeographischer Forschung, und war daher Richtstein für die Feld-Forschung und Schreibarbeit dieser Fallstudie.

\section{Raumaneignungen und Profite in Dhakas Feld des Straßenhandels}

Kaum eine andere Stadt auf der Welt wuchs in den vergangenen Dekaden so rapide wie die Hauptstadt Bangladeschs. In den 1950er Jahren lebten nicht einmal 400.000
Menschen in Dhaka, 60 Jahre später waren es etwa 15 Millionen (UN, 2012). Dieses schnelle Bevölkerungswachstum ging einher mit einer fragmentierenden Entwicklung, sowohl im sozio-ökonomischen, im kulturellen, im politischen, als auch im räumlichen Sinne (vgl. World Bank, 2007; Bertuzzo, 2009; Siddiqui et al., 2010). Aufgrund der extremen Unterschiede zwischen Einkommensklassen und Lebensstilen sollte eine „Raumaneignung von oben“, d.h. aus einer Position der Macht heraus, von einer subalternen „Raumaneignung von unten" unterschieden werden. Dhakas Eliten steuern die Stadt in ökonomischer und politischer Hinsicht und entwerfen die Pläne für die formale Stadtentwicklung, profitieren aber auch von informellen Raumaneignungen. So bauen Großinvestoren in der ganzen Stadt auf staatseigenen Flächen Wohnungsblöcke, Bürotürme und Kaufhäuser und lassen sich ihre illegale Landnahme durch ihre politischen Verbindungen nachträglich legalisieren. Sie eignen sich großflächig öffentliche Räume an, um Profite zu erzielen (vgl. Ghafur, 2004).

Im Gegensatz dazu wird die subalterne „Raumaneignung von unten“ von der „Macht der Notwendigkeit" angetrieben, wie es Bayat (1997:10) in einer Studie über Teheran beschreibt. Angesichts schlechter Zugangsmöglichkeiten zum Arbeitsmarkt und fehlender staatlicher Sozialsicherung eignen sich die Subalternen den städtischen Raum „schleichend“ oder „lautlos“ an (ebd.: 6). In Dhaka sind die ökonomisch und politisch Marginalisierten darauf angewiesen ihren Lebensunterhalt durch informelle wirtschaftliche Tätigkeiten zu sichern (vgl. Etzold et al., 2009; Kulke und Staffeld, 2009). So sind etwa 115.000 Menschen im Straßenhandel tätig; sie verkaufen Kleidung, Haushaltswaren, Zeitungen, und Nahrung. Unter ihnen sind etwa 97.000 Essensverkäufer (eigene Schätzung auf Grundlage amtlicher Beschäftigungsstatistiken und eigener Erhebungen; vgl. Etzold, 2013). Tagtäglich ignorieren sie das Gesetz, das sowohl den Verkauf von Essen im Freien als auch die Aneignung öffentlicher Räume verbietet, und besetzen Straßen, Gehwege, Parks und andere öffentlich zugängliche Plätze der Stadt (siehe Abbildung 2). Dadurch schaffen sie eine Lebensgrundlage für ihre eigenen Familien und tragen wesentlich zur Versorgung mit Nahrungsmitteln und somit letztlich zur Ernährungssicherung bei (Keck und Etzold, 2013).

Im Folgenden wird das Feld des Straßenhandels auf zwei unterschiedlichen Ebenen analysiert. Aus einer mikropolitischen Perspektive (vgl. Zimmer, 2012) stehen die Praktiken der Raumaneignung, das Spiel mit Regeln und territorialer Kontrolle, und die räumlichen Profite der beteiligten Akteure im Fokus. Es geht um die Akteure und Konflikte innerhalb des Feldes des Straßenhandels (Kapitel 4). Die Auseinandersetzungen um Straßenhändler und öffentliche Räume in Dhaka müssen hingegen auch im makro-politischen Kontext eingebettet werden. Es geht daher auch um die Diskurse über das Feld des Straßenhandels und seine Relation zum staatlich kontrollierten „Feld der Macht“ (Kapitel 5). 

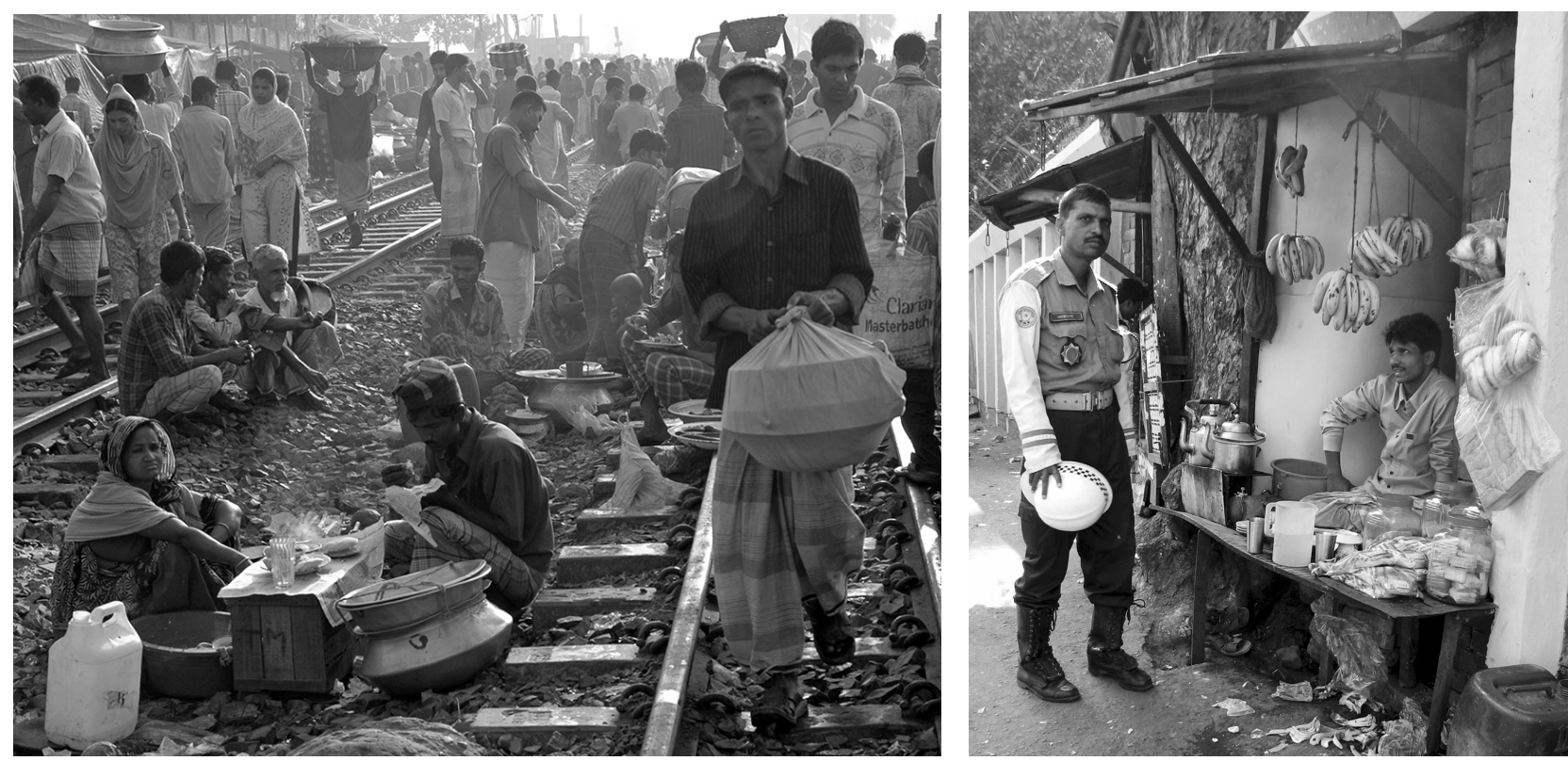

Abb. 2. Essen wird in der Megastadt Dhaka in jeder freien Nische verkauft. Mobile Essensverkäufer besetzen temporär sogar die Gleise der wichtigsten Zugverbindung in der Stadt (links). Ein Polizist kauft Tee und Snacks von einem kleinen permanenten Verkaufsstand am Straßenrand (rechts). (Fotos: B. Etzold, Kawran Bazar 02/2009, Shegunbagicha 01/2008)

\subsection{Raumaneignungen der Straßenhändler}

Straßenhändler besetzen nicht nur den physischen Raum Dhakas mit ihren Essensständen oder Handkarren, sondern nehmen auch Positionen im sozialen Raum der Stadt ein. Letztere sind wiederum maßgeblich für ihre Möglichkeiten, sich Verkaufsplätze anzueignen. Da die Temporalität bei der Raumaneignung eine wichtige Rolle spielt (Bourdieu, 2005b), wurde die große Bandbreite an Verkaufspraktiken in vier Mobilitäts-Typen zusammengefasst: Permanente Essensgeschäfte haben je nach Baumaterial einen mehr oder weniger großen materiellen und symbolischen Wert und somit auch eine mehr oder weniger große „Beharrungskraft“ (ebd. 118). Ein am Straßenrand mit Ziegelsteinen und Wellblech errichtetes kleines Restaurant, das Reisgerichte verkauft, ist ein dauerhafterer Ausdruck der Raumaneignung als ein einfacher aus Holz und Plastikfolie erbauter Tee-Stand über dem ansonsten offenen Abwasserkanal. Eine konsolidierte Bauweise erfordert allerdings eine gewisse rechtliche Absicherung des Verkaufsplatzes. Semi-permanente Verkaufsstände sind Handkarren oder Tische, die am Tag auf dem Gehweg aufgestellt werden. Nachts oder bei Bedarf, beispielsweise wenn eine Straßenräumung droht, können sie weg geschoben oder abgebaut werden. Von solchen Ständen werden Früchte, Getränke oder Backwaren angeboten. Mit semi-mobilen Verkaufsständen können Konsumenten an verschiedenen Orten erreicht werden. Auf manchen Rikschas sind Pressen installiert um Passanten frischen Zuckerrohrsaft zu verkaufen. Und um die Mittagszeit sitzen in ruhigeren Straßen Frauen auf dem Gehweg und servieren aus großen
Schüsseln einfache, zu Hause zubereitete Reisgerichte an Rikscha-Fahrer. Mobile Straßenhändler laufen im Gegensatz dazu mit einem Bauchladen voll Snacks, Süßigkeiten und Zigaretten, einem Korb voller Früchte oder einer Teekanne in belebten Straßen oder auf Plätzen umher. Sie eignen sich öffentliche Räume nur temporär an.

Wie bereits dargestellt vertritt Bourdieu (2005b:120) die These, dass die Fähigkeit den Raum zu beherrschen in erster Linie vom Kapitalbesitz abhängt. Eigene Untersuchungen belegen, dass die Verkaufsstrategie insbesondere vom ökonomischen Kapital abhängig ist, in erster Linie vom Vermögen des Straßenhändlers und seiner Familie. Ohne Sozialkapital hingegen haben die Straßenhändler keinen einfachen Zugang zu den Verkaufsstellen. So kann ein frisch in Dhaka eingetroffener Migrant nicht einfach an irgendeiner Straßenecke Essen verkaufen, denn alle Händler kennen sich untereinander und achten genau darauf, ob und wo ein neuer Verkäufer einen Stand eröffnet, und ob das ihren eigenen Geschäftserfolg beeinflusst. Eine permanente Raumaneignung ist daher nur durch Hilfe von Freunden, der Familie und lokalen Autoritäten möglich. Während nur vier Prozent der mobilen Verkäufer Hilfe beim Zugang zu ihrer regelmäßigen Verkaufsstelle erhielten, waren es 61 Prozent der permanenten Händler (vgl. Tabelle 1). Die mobilen Händler haben geringes Sozialkapital beziehungsweise haben sie kein Sozialkapital, welches ihnen eine dauerhafte Raumaneignung ermöglicht. Die permanenten Händler sind auf Grund ihrer jahrelangen Präsenz in der Arena besser in lokale Netzwerke eingebettet. Darüber hinaus besitzen sie gegenüber den anderen Verkaufsgruppen einen höheren sozialen Status (symbolisches 
Tabelle 1. Indikatoren für die soziale Position von Dhakas Straßenhändlern.

\begin{tabular}{|c|c|c|c|c|c|c|}
\hline \multirow{2}{*}{$\begin{array}{l}\text { Typen der } \\
\text { Raumaneignung }\end{array}$} & \multirow{2}{*}{$\begin{array}{l}\text { Anteil in } \\
\text { Studie }\end{array}$} & \multicolumn{3}{|c|}{ Ökonomisches Kapital } & \multirow{2}{*}{$\begin{array}{l}\text { Soziales Kapital } \\
\text { Anteil der Händler, } \\
\text { denen beim Zugang } \\
\text { zur Verkaufsstelle } \\
\text { geholfen wurde }\end{array}$} & \multirow{2}{*}{$\begin{array}{l}\text { Symbolisches } \\
\text { Kapital } \\
\text { Anteil der Händler, } \\
\text { die sich ggü. mo- } \\
\text { bilen Verkäufern als } \\
\text { „besser gestellt“ } \\
\text { betrachten }\end{array}$} \\
\hline & & $\begin{array}{l}\text { Leben unter } \\
\text { schlechten } \\
\text { Wohn- } \\
\text { bedingungen } \\
\text { (Anteil) }\end{array}$ & $\begin{array}{l}\text { Haushalts- } \\
\text { einkommen } \\
\text { pro Monat } \\
\text { (Durchschnitt } \\
\text { in BDT) }\end{array}$ & $\begin{array}{l}\text { Geschäfts- } \\
\text { gewinn pro } \\
\text { Monat (Durch- } \\
\text { schnitt in BDT) }\end{array}$ & & \\
\hline $\begin{array}{l}\text { Permanent } \\
\text { konsolidiert }\end{array}$ & $11 \%$ & $0 \%$ & 29.800 & 23.800 & $61 \%$ & $84 \%$ \\
\hline $\begin{array}{l}\text { Permanent } \\
\text { unkonsolidiert }\end{array}$ & $21 \%$ & $8 \%$ & 12.900 & 9.200 & $56 \%$ & $69 \%$ \\
\hline Semi-permanent & $13 \%$ & $13 \%$ & 19.900 & 8.900 & $20 \%$ & $33 \%$ \\
\hline Semi-mobil & $35 \%$ & $5 \%$ & 15.000 & 9.400 & $33 \%$ & $49 \%$ \\
\hline Mobil & $20 \%$ & $29 \%$ & 8.400 & 6.700 & $4 \%$ & $8 \%$ \\
\hline Gesamt & $100 \%$ & $11 \%$ & 15.400 & 10.300 & $33 \%$ & $45 \%$ \\
\hline nur Straßenhändler & $89 \%$ & $12 \%$ & 13.700 & 8.700 & $30 \%$ & $39 \%$ \\
\hline
\end{tabular}

Quelle: Eigene Feldforschung, November/Dezember $2009(n=120)$

Kapital). Sie werden von anderen als relativ besser gestellt betrachtet und sehen sich auch selbst so (vgl. Tabelle 1). Händler mit einer besseren Kapitalausstattung verfügen somit über größere Potentiale der Raumaneignung. Ihre Position im sozialen Raum ist zwar nicht mit der Position im physischen Raum identisch, dennoch ist ihr ,räumliches Kapital" ein bedeutender Indikator für ihre relativ gesehen bessere Position im Feld. Im Umkehrschluss bedeutet das allerdings nicht, dass die mobileren Händler machtlos sind. Vielmehr setzen sie das ihnen zu Verfügung stehende Kapital flexibel ein, um sich Raum temporär anzueignen. Sie schaffen durch ihre Bewegungen „Blasenräume“, die modernen stadtplanerischen Visionen und fest geordneten räumlichen Strukturen oftmals im Wege stehen (Redepenning et al., 2010:211).

\subsection{Raumaneignungen und das Spiel mit den Regeln in der Arena}

Um die Raumaneignungen der Straßenhändler zu verstehen, müssen sowohl gültige Gesetze, als auch informelle Spielregeln und alltägliche Aushandlungsprozesse an den Verkaufsorten betrachtet werden. Die Aneignung öffentlicher Räume, z.B. durch Errichten von Ständen auf Gehwegen, sowie die Zubereitung und der Verkauf von Nahrungsmitteln in nicht dafür explizit ausgewiesenen öffentlichen Räumen, wie Märkten, sind in Bangladesch seit der britischen Kolonialzeit per Gesetz verboten ${ }^{1}$. Bis heute gibt es für Straßenhändler keinerlei Möglichkeit, ihre zubereiteten Speisen, Snacks und

\footnotetext{
${ }^{1}$ Penal Code von 1860; Police Act von 1861; Pure Food Ordinance von 1959; Dhaka Metropolitan Police Ordinance von 1976; Dhaka City Corporation Ordinance von 1983.
}

Getränke auf öffentlichen Plätzen legal zu verkaufen. Aber die bloße Existenz solcher formeller Regeln und das ,,alltägliche Leben des Gesetzes“ (Anjaria, 2010) sind zwei unterschiedliche Dinge (vgl. Etzold, 2009; Etzold et al., 2012). Zum einen liegt das daran, dass das Gesetz nur bedingt in einer 15 Millionen-Stadt durchgesetzt werden kann, da mangelndes Personal oder überlappende Zuständigkeiten für verschiedene Räume auch zu unterschiedlicher „Wirksamkeit“ des Gesetzes führen. So ist die Universitätsverwaltung sehr viel strenger bei der Kontrolle des klar abgegrenzten Campus als die Stadtverwaltung, die für alle Straßen, Gehwege und Plätze im Stadtgebiet zuständig ist. Viele Straßenhändler nutzen unterschiedliche Zuständigkeiten der territorialen Kontrolle geschickt aus und verkaufen an Orten deren Regulation nicht eindeutig festgelegt ist oder ziehen sich in $\mathrm{Ni}$ schen jenseits staatlicher Kontrolle zurück.

Zum anderen sind staatliche Akteure, wie Beamte der Stadtverwaltung oder Polizisten, die den Straßenhandel per Gesetz nicht zulassen dürften, oftmals selbst zutiefst in die lokale informelle Politik eingebunden. Laut Bourdieu (2006) besitzen sie eine ,doppelte Macht“ auf Grund ihrer Position der Autorität, aus der sie auf formalem oder informellem Weg Profit schlagen können (vgl. Garcia-Rincon, 2007; Zimmer und Sakdapolrak, 2012). Wenn sie sich an festgeschriebene Regeln halten, können sie sich der Anerkennung ihrer Vorgesetzten als strenge Gesetzeshüter sicher sein. Wenn sie jedoch mit den Regeln spielen - denn das ,Spiel mit der Regel [ist] Teil der Regel des Spiels“ (Bourdieu, 1976:206) - indem sie hier und da ein Auge zudrücken, können sich korrupte Beamte diese „Ausnahme von der Regel“" vergüten lassen. In Bangladesch gehört der Missbrauch formaler Positionen 
durch Korruption und Klientelismus zur Tagesordnung. Städtische Governance in Dhaka ist geprägt durch enge Netzwerke, undurchsichtige Aushandlungsprozesse und Kriminalität (vgl. Siddiqui und Ahmed, 2004; World Bank, 2007). Und so sind Straßenverkäufer, Großhändler (Keck, 2012) oder Slumbewohner (Hossain, 2011; Hackenbroch, 2013) gezwungen, sich ihre Raumaneignungen, ihre Geschäfte oder ihren $\mathrm{Zu}-$ gang zu öffentlichen Dienstleistungen durch persönliche Beziehungen, politische Verbindungen und Schutzgeldzahlungen zu sichern.

Jenseits des Spiels mit der Formalität sind es informelle Regelungen, die für den Alltag der Straßenhändler die größte Relevanz haben. Der öffentliche Raum in Dhaka ist zwar für alle frei zugänglich, seine Nutzung oder ökonomische InWert-Setzung unterliegt jedoch einem dichten Netz an sozialen Normen und Regeln (vgl. Etzold et al., 2009; Hackenbroch, 2013). Die Essensverkäufer sprechen sich miteinander ab und unterstützen sich; allerdings nur wenn sich alle an die Spielregeln halten. So müssen alle Händler die Raumansprüche der anderen achten. Die etablierten Verkäufer können so die lukrativsten Plätze in der Arena behaupten, wohingegen Neuankömmlinge weitestgehend ,rechtlos“ sind; es sei denn sie haben gute persönliche Beziehungen zu lokalen Politikern, Geschäftsleuten oder Kriminellen. Denn diese „regieren“ den öffentlichen Raum in Dhaka und weisen den Händlern ,,ihren“ Verkaufsplatz zu. Im Feld des Straßenhandels gelten somit eigene Regeln, welche lokale Machtverhältnisse widerspiegeln und das Ergebnis von Aushandlungsprozessen sind. Diese informellen Governance-Modi werden härter sanktioniert als formale Gesetze, die nur lückenhaft durchgesetzt werden. Für Straßenhändler, und generell für viele der städtischen Armen, sind die informellen Zugangsund Nutzungsrechte zum öffentlichen Raum die entscheidende Grundlage der alltäglichen Lebenssicherung (vgl. Etzold, 2013; Hackenbroch et al., 2009; Hackenbroch, 2013).

\subsection{Raumprofite im Feld des Straßenhandels}

Durch die Aneignung spezifischer Orte, durch die ,richtige Adresse" in einem hoch angesehenen Wohnviertel oder durch die Nähe zu begehrten Dienstleistungen, d.h. durch die Verfügungsgewalt über den physischen Raum, können Akteure „Raumprofite“ erzielen (Bourdieu, 1991). Art und Umfang der Raumprofite sind Ausdruck der Position im Feld und somit auch bestehender Machtverhältnisse (Schroer, 2006). Im Folgenden wird gezeigt, welche Gewinne die Essensverkäufer, ihre Kunden und die informellen Regulatoren öffentlicher Räume aus der subversiven Raumaneignung der Straßenhändler erzielen.

\subsubsection{Raumprofite der Straßenhändler}

Insofern sie die gültigen Spielregeln beherrschen, können sich Straßenhändler die öffentlichen Räume Dhakas aneignen, jedoch in unterschiedlichem Maße. Tabelle 1 zeigt, dass
Umfang und Dauer der Raumaneignung in direktem Verhältnis zur Einkommens- und Lebenssituation der Essensverkäufer, sprich zu ihren Raumprofiten und ihrer Position im sozialen Raum, stehen. So wohnt knapp ein Drittel der mobilen Straßenhändler unter sehr schlechten Bedingungen in Slums oder sogar auf der Straße, wohingegen kein Besitzer einer baulich-konsolidierten Imbissbude unter schlechten Wohnverhältnissen leidet ${ }^{2}$. Die Unterschiede im Haushaltseinkommen und dem Geschäftsgewinn sind erheblich. Mobile Straßenhändler verdienen mit umgerechnet 6.710 Bangladeschischen Taka ${ }^{3}$ im Monat am wenigsten; ihren Haushalten steht auch am wenigsten Geld zur Verfügung. Die semi-permanenten Händler verdienen mit 8.900 Taka im Monat zwar nicht sehr viel mehr, da andere Familienmitglieder tendenziell aber auch hinzuverdienen, verfügen sie über ein mehr als doppelt so hohes Haushaltseinkommen. Mit einem monatlichen Gewinn von 23.800 Taka sind die Besitzer der konsolidierten Geschäfte im Vergleich zu den eigentlichen Straßenhändlern schon relativ wohlhabend. Das skizzierte Kontinuum an Praktiken der Raumaneignung bildet auch ein Gefälle der sozialen Verwundbarkeit im Feld des Straßenhandels ab (vgl. Etzold et al., 2012).

\subsubsection{Raumprofite der Street Food Konsumenten}

Die Verkäufer sind natürlich nicht die Einzigen, die von ihrer Aneignung öffentlicher Räume profitieren. Die Konsumenten, potentiell etwa die Hälfte der Einwohner Dhakas, haben enorme Vorteile davon, dass es zubereitetes Essen, frisches Obst und Getränke an nahezu jeder Straßenecke gibt. Das Essen von der Straße ist überall dort verfügbar wo viele Menschen arbeiten, durchreisen oder ihre Freizeit verbringen aus Bourdieus (1991) Perspektive wären dies „Lokalisationsprofite“. Darüber hinaus ist Street Food auf Grund der niedrigeren Betriebskosten billiger als Gerichte aus registrierten Imbissbuden und Restaurants, und sogar günstiger als zu Hause gekochtes Essen, wenn man die benötigte Zeit und Kosten für Einkauf und Zubereitung miteinberechnet. Des Weiteren genießen es viele Menschen bei einer Tasse Tee auf einem Platz zu diskutieren, oder mit ihren Kindern im Park zu sein und Snacks zu essen (vgl. Etzold, 2013). Der gesellschaftliche und ökonomische Mehrwert von Street Food bemisst sich somit nicht nur am Beitrag zur Lebenssicherung von städtischen Armutsgruppen (Tinker, 1997) und zur urbanen Ernährungssicherung (Keck und Etzold, 2013), sondern eben auch an der Belebung des öffentlichen Lebens (Bertuzzo, 2009). Durch ihre Raumaneignungen ermöglichen die Straßenhändler diese indirekten Raumprofite.

\footnotetext{
${ }^{2}$ Die permanenten und baulich konsolidierten Essensstände sind in Tabelle1 als Vergleichsgruppe aufgeführt, denn sie gehören nicht zum Straßenhandel. Laut Definition von Tinker (1997:17) sind Street Food-Geschäfte solche, die fertig zubereitetes Essen mobil oder von einer Struktur mit weniger als drei Wänden verkaufen.

${ }^{3}$ Das entspricht etwa 64 EUR; Wechselkurs am 1. Dezember 2009: 100 Bangladeschischer Taka $(B D T)=0,95$ EUR.
} 


\subsubsection{Raumprofite der mächtigen Akteure in der Arena}

Am meisten profitieren allerdings jene lokal mächtigen Akteure, die die geltenden Gesetze und informellen Regeln im öffentlichen Raum kontrollieren und durchsetzen. In Dhaka müssen sich die Geschäfte ihre Sicherheit durch Schutzgeldzahlungen erkaufen. Machen sie das nicht, so müssen die Händler damit rechnen von kriminellen Akteuren aus ihrem Laden vertrieben zu werden oder ihnen wird Gewalt angedroht um die fälligen Beträge zu erzwingen (vgl. Siddiqui und Ahmed, 2004; World Bank, 2007; Keck, 2012). Auch die Straßenhändler erwerben mit so genannten Chanda-Zahlungen das „Recht“ zur Aneignung ihrer Nische im öffentlichen Raum. Das Zahlen solch informeller Nutzungsgebühren gehört für die Essensverkäufer zum Alltag und wird von ihnen als normaler, wenn auch ärgerlicher Teil der Geschäftsausgaben veranschlagt. Die meisten Händler zahlen zwischen 10 und 500 Taka am Tag an lokale Vermittler (so genannte Linesmen), die den Großteil des Geldes an vor Ort mächtige Kriminelle (so genannte Mastaans), an lokale Politiker und an Polizisten weiterreichen. Die Höhe der fälligen Zahlung hängt vom Umsatz und der Größe der Straßenstände ab sowie von der Attraktivität ihres Verkaufsplatzes. Basierend auf eigenen Hochrechnungen werden in Dhaka jährlich etwa 3,4 Milliarden Taka (etwa 32,6 Millionen Euro) illegal von den Straßenhändlern erpresst (Etzold, 2013). Die lokal tätigen Polizisten, Bürokraten und Politiker erzielen ihre „Positions- oder Rangprofite“ (Bourdieu, 1991) aus ihrer Position an einer Schnittstelle zwischen dem Feld der Staatsmacht und dem Feld des Straßenhandels und ihrer ,,doppelten Macht" der Durchsetzung oder Umgehung des Rechts (Bourdieu, 2006). Die Mittelsmänner und Kriminellen profitieren durch ihre Kontakte zu Politikern und Polizisten und durch die Anerkennung ihrer Position durch die Straßenhändler. Schutzgeldzahlungen sind der unliebsame Preis der subalternen Raumaneignung und letztlich Ausdruck der asymmetrischen Machtverhältnisse im Feld des Straßenhandels.

\section{Das Feld des Straßenhandels im Schatten des „Feldes der Macht"}

Die bisherigen Ausführungen haben gezeigt, dass Lebenschancen, Raumprofite und Machtpotentiale innerhalb des Feldes des Straßenhandels höchst ungleich verteilt sind. Die relative Bedeutung des Straßenhandels in der Stadt, und somit die Position dieses spezifischen Feldes zum staatlich kontrollierten „Feld der Macht“, lässt sich auf einer diskursiven Ebene gut darstellen. Warum wird das Feld des Straßenhandels in Bangladesch so gering geschätzt? Erstens scheinen Straßenhändler auf Grund ihrer guten Sichtbarkeit im öffentlichen Raum „,beliebte Sündenböcke“ zu sein, die der Elite und aufstrebenden Mittelschicht eine Projektionsfläche für ihre moderne Raumideologie einer sauberen und ästhetisch gestalteten Stadt frei von Störungen (durch die Marginalisierten) bieten. So betonen Behördenmitarbeiter, Polizi- sten, Stadtplaner und auch Journalisten immer wieder, dass der Verkauf von Street Food nicht nur gesetzeswidrig, sondern auch chaotisch, unästhetisch und unhygienisch sei. Die Straßenverkäufer stünden einer ,modernen“Entwicklung der Stadt, die gekennzeichnet ist durch den Bau von Büro- und Wohntürmen sowie klimatisierten Einkaufszentren mit FastFood-Shops, im wahrsten Sinn des Wortes ,im Wege“ (vgl. Cross und Karides, 2007; Redepenning et al., 2010) ${ }^{4}$. Eine Medienanalyse von Artikeln einer lokalen Tageszeitung ergab, dass die negativen Narrative über Straßenhändler und Street Food die eher positiven Darstellungen deutlich überwiegen ${ }^{5}$. Zweitens scheint die Bekämpfung des Straßenhandels ein „Ventil““ zur Entladung politischen Drucks zu sein, insbesondere in Krisenzeiten. Die Vertreibung von Straßenhändlern und die Zerstörung von illegal errichteten Bauten waren im Januar und Februar 2007 die ersten wirkungsvollen und öffentlich sichtbaren Maßnahmen der so genannten Übergangsregierung in Bangladesch. Während dieser zweijährigen Staatskrise (2007/08) fanden wiederholt umfangreiche Polizeiaktionen zur „Rückeroberung des öffentlichen Raumes" statt. Die Vertreibung von ihren Verkaufsstellen wurde für viele Straßenhändler zur existentiellen Bedrohung. Den staatlichen Akteuren ging es dabei nicht nur um die Zurückweisung von als illegitim erachteten Raumansprüchen, sondern vor allem auch um die sichtbare Wiederherstellung von „Recht und Ordnung“ und die Demonstration ihrer Handlungsfähigkeit und Autorität (vgl. Etzold, 2013).

Die Machtbeziehungen zwischen dem Feld des Straßenhandels und dem „Feld der Macht“ zeigen sich somit auf zwei Ebenen. Auf der makro-politischen Ebene werden die alltäglichen Raumaneignungen von Straßenhändlern durch das Gesetz kriminalisiert, durch staatliche Kampagnen und Räumungsaktionen bekämpft und mit Hilfe der Diskurse des Rechts, der modernen Stadt, der öffentlichen Sicherheit und der Hygiene delegitimiert. Der Beitrag der Straßenverkäufer

\footnotetext{
${ }^{4}$ In Interviews mit Vertretern der Stadtverwaltung, eines Krankenhauses, der Dhaka University, der Polizei und des Think Tanks Centre for Urban Studies waren die hygienischen Bedingungen von Street Food, die Konkurrenz des Straßenhandels mit anderen Nutzungen des öffentlichen Raumes, aber auch die Notwendigkeit des Straßenhandels als Einkommensquelle für die Armen immer wiederkehrende Themen (vgl. Etzold, 2013).

${ }^{5}$ Medienanalyse von 210 Artikeln aus der Tageszeitung „The Daily Star“ im Zeitraum 2003 bis 2009. Die den Straßenhandel eher ablehnenden Diskurse überwiegen in der medialen Darstellung: Narrative über eine funktionierende Stadt wurden 69 mal genannt, solche über eine moderne Stadt 19 mal, über Sicherheit im öffentlichen Raum 14 mal, über Hygiene 39 mal, über Illegalität 31 mal, und über Korruption 52 mal (insges. $72 \%$ aller Nennungen waren eher negativ). Positive Aspekte fallen deutlich weniger ins Gewicht (28\% aller Nennungen): der Nutzen des Straßenhandels im Sinne einer Nahrungsmittelversorgung wurde 33 mal genannt, die Bedeutung für die städtische Lebenssicherung und Armutsbekämpfung wurde 36 mal aufgegriffen und 19 mal wurde Street Food als wichtiges Element der lokalen Essenskultur hervorgehoben (vgl. Etzold, 2013).
} 
zur Nahrungsversorgung, zum Funktionieren der Stadt sowie zur Qualität des urbanen Lebens wird von den Behörden, den politischen Machthabern und den wirtschaftlichen Eliten hingegen nicht anerkannt. Auf der mikro-politischen Ebene wird es allerdings weitestgehend toleriert, dass Straßenverkäufer ihre Waren auf Gehwegen anbieten. In den lokalen Arenen lassen einige staatliche Akteure den Straßenhandel zu, um durch informelle Nutzungsgebühren substantielle Raumprofite zu erzielen. Darüber hinaus werden auf dieser Alltagsebene andere Diskurse eingesetzt: man kennt und unterstützt sich, und Polizisten und Wachmänner drücken auch mal ein Auge zu, um den vermeintlich armen Straßenhändlern ein Auskommen zu ermöglichen (vgl. Etzold et al., 2012). Solche Widersprüche zwischen den Handlungen und den Diskursen auf der Mikro-Ebene des Alltagslebens und der Makro-Ebene der städtischen Politik sind kennzeichnend für das Feld des Straßenhandels, das in den Megastädten des globalen Südens „,bedroht, verdrängt, und doch gebraucht“ (Dittrich, 2008) wird.

\section{Fazit}

Es war Ziel dieses Beitrages den Zusammenhang zwischen der Position von Dhakas Straßenhändlern in ihrer Arena, im Sinne ihres angeeigneten Verkaufsplatzes im öffentlichen Raum, und ihrer Position im „Feld des Straßenhandels“ im Sinne ihres Geschäftserfolgs und ihrer gesellschaftlichen Anerkennung, zu erörtern. Mit Bourdieus Theorie kann diese Dialektik von sozialer und räumlicher Positionierung angemessen erfasst werden. Darüber hinaus bieten sich Anknüpfungspunkte zu aktuellen Diskussionen um Raumaneignungen und Informalität in Städten des Globalen Südens, beispielsweise im Sinne von postkolonialen Konzepten des subaltern urbanism (vgl. Roy, 2011). Die Aktualität und Anschlussfähigkeit von Bourdieus Theorie lässt sich anhand von zwei abschließenden Thesen zeigen.

These 1: Aufgrund ihrer marginalen sozialen Position besetzen subalterne Akteure die übrig geblieben räumlichen „Nischen“ in der Stadt. Die informelle Aneignung des städtischen Raumes durch subalterne Akteure, wie Slumbewohner oder Straßenhändler, kann als Überlebensstrategie, aber auch als Ausdruck kollektiven Widerstandes gegen staatliche Willkür und Vernachlässigung verstanden werden. In der „Raumaneignung von unten“ zeigt sich die Dialektik von Handlungszwang, auf Grund der „Macht der Notwendigkeit“ (Bayat, 1997:10), und Handlungsfreiheit, im Sinne des Erkämpfens eines ,Rechts auf Stadt“ (u.a. Holm und Gebhardt, 2011). Subalterne Raumaneignungen sind ein immanenter Bestandteil des „Habitus der Enteigneten“ (Bayat, 2007). Das vorgestellte Fallbeispiel bestätigt diese Annahmen. Dhakas Straßenhändler haben ihre marginale Position verinnerlicht. Sie wissen, dass ihr Besetzen und In-Wert-Setzen öffentlicher Räume per Gesetz verboten ist. Daher legitimieren sie ihre ,illegalisierte“ Handlung auf Grund ihrer Bedürf- tigkeit und der hohen Nachfrage nach ihren Dienstleistungen. Ihre Raumaneignung ist alltägliche Routine, erfordert allerdings auch Auseinandersetzungen mit mächtigeren Akteuren, welche die Verkaufsplätze de facto ,regieren“. Mit Bourdieu gesprochen manifestiert sich so die soziale Position der Händler im physischen Raum. Sie besetzen übrig gebliebene „Freiräume“ - öffentliche Plätze, Straßenränder und Gehwege. Die „feinen Unterschiede“ innerhalb des Feldes des Straßenhandels drücken sich wiederum in unterschiedlichen Potentialen der Raumaneignung, ihrer mehr oder weniger großen „Beharrungskraft“ und den realisierten Raumprofiten aus.

These 2: Die Raumaneignungen der subalternen Akteure werden von staatlichen Akteuren ,informalisiert“ und kriminalisiert, um bestehende Machtasymmetrien und die ungleiche Verteilung von Raum und Kapital aufrecht zu erhalten. Seit über 40 Jahren wird Informalität im akademischen Diskurs auf den ,,informellen Sektor“ der städtischen Armen verkürzt, wohingegen die informellen Praktiken, Raumaneignungen und Bereicherungsstrategien der Eliten vernachlässigt werden. Informalität ist aber kein gesellschaftliches Randphänomen. Vielmehr sind informelle Aushandlungsprozesse und persönliche Absprachen im urbanen Süden oftmals wirkungsmächtiger, verlässlicher und dauerhafter als Gesetzestexte und bürokratische Praktiken (Roy und Alsayyad, 2004; Roy, 2011). In Dhaka eignen sich die Mächtigen tagtäglich Raum auf informelle Weise an. Durch politische Beziehungen werden die dadurch gewonnenen Raumprofite nachträglich legalisiert. Im Gegensatz dazu werden die alltäglichen Praktiken und Raumaneignungen der Armen kriminalisiert und delegimiert, um die legitimen Forderungen der Subalternen auf ihr ,Recht auf Stadt“ zurückzuweisen. In Dhaka zeigt sich genau dieses Muster im öffentlichen Diskurs: Die Straßenverkäufer agierten illegal, seien anderen Nutzungen im Weg und gefährdeten das Bild einer modernen Großstadt. Darüber hinaus sei Street Food unhygienisch und gesundheitsgefährdend. Staatliche Akteure bedienen sich solcher Narrative, um die Vertreibung von Essensverkäufern und die ,Verschönerungen“ öffentlicher Räume zu rechtfertigen. Sie stellen so die Lebenssicherung der Subalternen in Frage. Gleichzeitig werden die subalternen Raumaneignungen der Straßenhändler aber auch von lokalen Politikern und Polizisten toleriert. Aus der Perspektive der Theorie der Praxis sind diese Erkenntnisse nicht überraschend, denn durch ihre Nähe zum „Feld der Macht“ können staatliche Akteure auf formalen und informellen Wegen Kapitalgewinne erzielen. Und so sind Dhaka's öffentliche Räume keineswegs chaotisch oder unreguliert, auch wenn nicht formale Gesetze, sondern informelle Regeln die Raumaneignungen der Straßenhändler strukturieren (vgl. Etzold et al., 2009). Laut Roy (2011:233) führt diese „Informalisierung“ zur weiteren Benachteiligung von marginalisierten Bevölkerungsgruppen und verstärkt so bestehende Ungleichheiten.

Um soziale Ungleichheit, Raumaneignungen und Lebenssicherungsstrategien im urbanen Süden zu untersuchen, muss 
man sich natürlich nicht Bourdieus Theorie bedienen. Andere konzeptionelle Zugänge, wie Lefebvres Theorie der Raumproduktion (u.a. Bertuzzo, 2009), De Certeaus Theorie des Alltagslebens (u.a. Rothfuß, 2012), die angesprochenen postkolonialen Ansätze des subaltern urbanism (u.a. Roy, 2011) oder auch Grounded Theory (u.a. Hackenbroch, 2013) scheinen ebenso geeignet. Wo liegt dann der Mehrwert einer praxeologischen Perspektive für die sozialgeographische Stadtforschung? Die Stärke der Theorie der Praxis liegt meines Erachtens darin, dass sie relationale, kritische und reflexive Elemente in sich kohärent vereint und so einen „holistischen Blick“ auf die Räumlichkeit von Gesellschaft ermöglicht. Aus einer relationalen Perspektive stehen Netzwerke, Abhängigkeiten, Interaktionen und Raumaneignungen von Akteuren innerhalb eines Feldes sowie die Beziehungen zwischen verschieden Feldern und Arenen - eben die gesellschaftlichen und räumlichen Relationen - im Zentrum der Analyse. Eine kritische Sozialgeographie versucht, die in gesellschaftlichen Strukturen, im Alltagshandeln und im Raum eingeschriebene Machtverhältnisse zu erkennen und zu erklären - sowohl auf der makro-politischen Ebene der Gesetze und Diskurse als auch der mikro-politischen Ebene der Alltagspraktiken und Aushandlungsprozesse. Eine reflexive Sozialgeographie hinterfragt die Praxis der Wissensproduktion, die Positionierungen der forschenden und beforschten Subjekte, die eingesetzten Methoden sowie vollzogene Kategorisierungen und Bedeutungszuschreibungen. Gerade diese Aspekte werden momentan in der Geographischen Entwicklungsforschung im Kontext der Postkolonialismus-Debatte und der post-development Kritik diskutiert (vgl. u.a. MüllerMahn und Verne, 2010; Neuburger und Schmitt, 2012; Deffner und Haferburg, in dieser Ausgabe).

Danksagung. Ich möchte mich bei Dhakas Straßenhändlern bedanken; sie haben mich in vielen Stunden an ihrem Leben teilhaben lassen. Der Deutschen Forschungsgemeinschaft danke ich für die finanzielle Förderung des Projektes „,The Megaurban Food System of Dhaka“ (BO 680/35-1/-2) im Rahmen des Schwerpunktprogrammes „Megacities - Megachallenges“ (SPP 1233). Des Weiteren bedanke ich mich herzlich bei Hans-Georg Bohle (Universität Bonn), Wolfgang-Peter Zingel (Universität Heidelberg), Shafique uz Zaman (University of Dhaka) und meinen Forschungsassistenten, insbesondere Taufique Hassan, Sania Rahman und Afjal Hossain, sowie den Koordinatoren und Kollegen im SPP für die Zusammenarbeit. Patrick Sakdapolrak, Felix Hewel sowie den Herausgebern und Reviewern der Geographica Helvetica danke ich für zahlreiche konstruktive Anregungen.

Edited by: B. Korf

Reviewed by: four anonymous referees

\section{Literatur}

Anjaria, J.: The Politics of Illegality: Mumbai Hawkers, Public Space and the Everyday Life of the Law, in: Street Vendors in the Global Urban Economy, edited by: Bhowmik, S. K., Routledge, New Delhi/Abingdon, 69-86, 2010.

Bayat, A.: Street Politics: Poor People's Movements in Iran, Columbia University Press, New York, 1997.

Bayat, A.: Radical Religion and the Habitus of the Dispossessed: Does Islamic Militancy Have an Urban Ecology?, Int. J. Urban Regional, 31, 579-590, 2007.

Belina, B., und Michel, B.: Raumproduktionen. Zu diesem Band, in: Raumproduktionen - Beiträge der Radical Geography. Eine Zwischenbilanz, 2. Aufl. ed., Herausgeber: Belina, B. und Michel, B., Westfälisches Dampfboot, Münster, 7-34, 2008.

Bertuzzo, E. T.: Fragmented Dhaka. Analysing everyday life with Henri Lefebvre's Theory of Production of Space, Sozialgeographische Bibliothek, Band 10, Herausgeber: Werlen, B., Franz Steiner Verlag, Stuttgart, 2009.

Bhowmik, S. K.: Street Vendors in the Global Urban Economy, Routledge, New Delhi/Abingdon, 2010.

Bohle, H.-G. und Sakdapolrak, P.: Leben mit der Krise. Vertreibung von Slumbewohnern in der Megastadt Chennai, Geographische Rundschau, 60, 12-21, 2008.

Bourdieu, P.: Entwurf einer Theorie der Praxis auf der ethnologischen Grundlage der kabylischen Gesellschaft, Suhrkamp, Frankfurt am Main, 1976.

Bourdieu, P.: Physischer, sozialer und angeeigneter physischer Raum, in: Stadt-Räume, Herausgeber: Wentz, M., Campus, Frankfurt a.M., 25-34, 1991.

Bourdieu, P.: Praktische Vernunft: Zur Theorie des Handelns, Suhrkamp, Frankfurt am Main, 1998.

Bourdieu, P.: Verstehen, in: Das Elend der Welt. Gekürzte Studienausgabe, Herausgeber: Bourdieu, P., Balazs, G., Beaud, S., Broccolichi, S., Champagne, P., Christin, R., Lenoir, R., Oevrard, F., Pialoux, M., Sayad, A., Schultheis, F., und Soulie, C., UVK, Konstanz, 393-410, 2005a.

Bourdieu, P.: Ortseffekte, in: Das Elend der Welt. Gekürzte Studienausgabe, Herausgeber: Bourdieu, P., Balazs, G., und Beaud, S., UVK, Konstanz, 117-123, 2005b.

Bourdieu, P.: Das Recht und die Umgehung des Rechts, in: Pierre Bourdieu: Neue Perspektiven für die Soziologie der Wirtschaft, Herausgeber: Florian, M. und Hillebrandt, F., VS Verlag, Wiesbaden, 19-41, 2006.

Bourdieu, P. und Wacquant, L. J. D.: Die Ziele der reflexiven Soziologie, in: Reflexive Anthropologie, Herausgeber: Bourdieu, P. und Wacquant, L. J. D., Suhrkamp, Frankfurt am Main, 95-249, 1996.

Brown, A.: Contested Space. Street Trading, Public Space, and Livelihoods in Developing Cities, ITDG Publishing, Rugby, 2006.

Cross, J. und Karides, M.: Capitalism, modernity, and the "appropriate" use of space, in: Street Entrepeneurs. People, place and politics in local and global perspective, Herausgeber: Cross, J. und Morales, A., Routledge, London/New York, 19-35, 2007.

Davis, M.: Planet of Slums, Verso, London/New York, 2007.

Dittrich, C.: Bedroht, verdrängt und doch gebraucht. Der mobile Kleinhandel mit Nahrungsmitteln in der indischen Metropole Hyderabad, Geographische Rundschau, 60, 22-29, 2008. 
Dörfler, T., Graefe, O., and Müller-Mahn, D.: Habitus und Feld : Anregungen für eine Neuorientierung der geographischen Entwicklungsforschung auf der Grundlage von Bourdieus "Theorie der Praxis", Geogr. Helv., 58, 11-23, doi:10.5194/gh-58-112003, 2003.

Dörfler, T.: Gentrification in Prenzlauer Berg? Milieuwandel eines Berliner Sozialraums seit 1989, Transcript, Bielefeld, 2010.

Etzold, B.: Die umkämpfte Stadt - Die alltägliche Aneignung öffentlicher Räume durch Straßenhändler in Dhaka (Bangladesch), in: Initiativen für ein Recht auf Stadt: Theorie und Praxis städtischer Aneignungen, Herausgeber: Holm, A. und Gebhardt, D., VSA Verlag, Hamburg, 187-220, 2011.

Etzold, B.: The Politics of Street Food. Contested Governance and Vulnerabilities in Dhakas Field of Street Vending, Megacities and Global Change, Band 13, Franz Steiner Verlag, Stuttgart, 2013.

Etzold, B., Keck, M., Bohle, H.-G., und Zingel, W.-P.: Informality as Agency. Negotiating Food Security in Dhaka, Die Erde, 140, 3-24, 2009.

Etzold, B., Jülich, S., Keck, M., Sakdapolrak, P., Schmitt, T., und Zimmer, A.: Doing Institutions. A Dialectic Reading of Institutions and Social Practices and its Relevance for Development Geography, Erdkunde, 66, 185-195, 2012.

Frey, O.: Urbane öffentliche Räume als Aneignungsräume. Lernorte eines konkreten Urbanismus?, in: "Aneignung” als Bildungskonzept der Sozialpädagogik, Herausgeber: Deinet, U. und Reutlinger, C., VS Verlag, Wiesbaden, 219-233, 2004.

Garcia-Rincon, M. F.: Redefining rules: a market for public space in Caracas, Venezuela, in: Street Entrepeneurs. People, place and politics in local and global perspective, Herausgeber: Cross, J. und Morales, A., Routledge, London/New York, 36-57, 2007.

Ghafur, S.: Social Exclusion and Residential Densification: Implications for Integration of the Urban Poor in Dhaka, Bangladesh, Protibesh, BUET, 52-54, 2004.

Glasze, G., Pütz, R., und Rolfes, M.: Diskurs - Stadt - Kriminalität. Städtische (Un)Sicherheiten aus der Perspektive von Stadtforschung und Kritischer Kriminalgeographie, Urban Studies, transcript, Bielefeld, 2005.

Graefe, O. and Hassler, M.: Aktuelle Ansätze einer relationalen Humangeographie in Entwicklungsländern : Enführung zum Themenheft, Geogr. Helv., 61, 2-3, doi:10.5194/gh-61-2-2006, 2006.

Hackenbroch, K.: The Spatiality of Livelihoods: Negotiations of Access to Public Space in Dhaka, Bangladesh, Megacities and Global Change, Band 7, Franz Steiner Verlag, Stuttgart, 2013.

Hackenbroch, K., Baumgart, S., und Kreibich, V.: Spatiality of Livelihoods: Urban Public Space and the Urban Poor in Dhaka, Die Erde, 140, 47-68, 2009.

Holm, A. und Gebhardt, D.: Initiativen für ein Recht auf Stadt: Theorie und Praxis städtischer Aneignungen, VSA Verlag, Hamburg, 2011.

Hossain, S.: Informal Dynamics of a Public Utility: Rationality of the Scene behind a Screen, Habitat International, 35, 275-285, 2011.

Keck, M.: Informality as borrowed security: Contested food markets in Dhaka, Bangladesh, in: Urban Informalities. Reflections on the Formal and Informal, Herausgeber: Waibel, M. und McFarlane, C., Ashgate, London, 111-127, 2012.
Keck, M. und Etzold, B.: Resilience Refused - Wasted Potentials for Improving Food Security in Dhaka, Erdkunde, 67, 75-91, 2013.

Klamt, M.: Öffentliche Räume, in: Handbuch Stadtsoziologie, Herausgeber: Eckhardt, F., Springer, Wiesbaden, 775-804, 2012.

Kühne, O.: Kritische Geographie der Machtbeziehungen. Konzeptionelle Überlegungen auf der Grundlage der Soziologie Pierre Bourdieus, Geographische Revue, 10, 40-50, 2008.

Kulke, E. und Staffeld, R.: Informal Production Systems - The Role of the Informal Economy in the Plastic Recycling and Processing Industry in Dhaka, Die Erde, 140, 25-43, 2009.

Lippuner, R.: Reflexive Sozialgeographie. Bourdieus Theorie der Praxis als Grundlage für sozial- und kulturgeographisches Arbeiten nach dem cultural turn, Geogr. Z., 93, 135-147, 2005a.

Lippuner, R.: Raum - Systeme - Praktiken. zum Verhältnis von Alltag, Wissenschaft und Geographie, Sozialgeographische Bibliothek, Herausgeber: Werlen, B., Franz Steiner Verlag, Stuttgart, 2005 b.

Lossau, J. und Lippuner, R.: Geographie und Spatial Turn, Erdkunde, 58, 201-211, 2004.

Low, S. M. und Smith, N.: The Politics of Public Space, Routledge, Abingdon/New York, 200 pp., 2006.

Madanipour, A.: Marginale öffentliche Räume in europäischen Städten, DISP, 155, 4-17, 2003.

Madanipour, A.: Whose Public Space? International Case Studies in Urban Design and Development, Routledge, London, New York, 2010.

Mitchell, D.: The Right to the City: Social Justice and the Fight for Public Space, The Guilford Press, New York, 2003.

Müller-Mahn, D. und Verne, J.: Geographische Entwicklungsforschung - alte Probleme, neue Perspektiven, Geographische Rundschau, 62, 4-11, 2010.

Neuburger, M. and Schmitt, T.: Editorial Theorie der Entwicklung - Entwicklung der Theorie Post-Development und Postkoloniale Theorien als Herausforderung für eine Geographische Entwicklungsforschung, Geogr. Helv., 67, 121-124, doi:10.5194/gh-67121-2012, 2012.

Redepenning, M., Neef, H., and Gomes, E.: Verflüssigende (Un-) Sicherheiten : über Räumlichkeiten des Strassenhandels am Beispiel Brasiliens, Geogr. Helv., 65, 207-216, doi:10.5194/gh-65207-2010, 2010.

Rothfuß, E.: Intersubjectivity, Intercultural Hermeneutics and the Recognition of the Other - Theoretical Reflections on the Understanding of Alienness in Human Geography Research, Erdkunde, 63, 173-188, 2009.

Rothfuß, E.: Exklusion im Zentrum. Die brasilianische Favela zwischen Stigmatisierung und Widerständigkeit, Transcript, Bielefeld, 2012.

Roy, A.: Slumdog Cities: Rethinking Subaltern Urbanism, Int. J. Urban Regional, 35, 223-238, 2011.

Roy, A. und Alsayyad, N.: Urban Informality. Transnational Perspectives from the Middle East, Latin America, and South Asia, Lexington Books, Oxford, 2004.

Sakdapolrak, P.: Orte und Räume der Health Vulnerability. Bourdieus Theorie der Praxis für die Analyse von Krankheit und Gesundheit in megaurbanen Slums von Chennai, Südindien, Studies in Development Geography, Band 38, Verlag für Entwicklungspolitik, Saarbrücken, 2010. 
Schroer, M.: Räume, Orte, Grenzen. Auf dem Weg zu einer Soziologie des Raumes, 1, Suhrkamp, Frankfurt a.M., 2006.

Siddiqui, K., Ahmed, J., Siddiqui, K., Huq, S., Hossain, A., Nazimud-Doula, S., und Rezawana, N.: Social Formation in Dhaka, 1985-2005. A Longitudinal Study of Society in a Third World Megacity, Ashgate, Surrey, 2010.

Siddiqui, K. und Ahmed, J.: Dhaka, in: Megacity Governance in South Asia. A Comparative Study, Herausgeber: Siddiqui, K., Ghosh, A., Bhowmik, S. K., Siddiqi, S. A., Mitra, M., Kapuria, S., Ranjan, N., und Ahmed, J., University Press Limited, Dhaka, 353-432, 2004.

Tinker, I.: Street Foods: Urban Food and Employment in Developing Countries, Oxford University Press, New York/Oxford, 1997.

UN: World Urbanization Prospects: The 2011 Revision. Highlights, United Nations Department of Economic and Social Affairs, Population Division, New York, 2012.

Wacquant, L. J. D.: Auf dem Weg zu einer Sozialpraxeologie. Struktur und Logik der Soziologie Pierre Bourdieus, in: Reflexive Anthropologie, Herausgeber: Bourdieu, P. und Wacquant, L. J. D., Suhrkamp, Frankfurt am Main, 17-93, 1996.
Werlen, B.: Sozialgeographie alltäglicher Regionalisierungen. Band 2: Globalisierung, Region und Regionalisierung, 2.überarb. Aufl. ed., Erdkundliches Wissen, H. 119, Steiner Verlag, Stuttgart, 1997.

Werlen, B. und Lippuner, R.: Sozialgeographie, in: Geographie. Physische und Humangeographie, Herausgeber: Gebhardt, H., Glaser, R., Radtke, U., und Reuber, P., Elsevier/Spektrum Akademischer Verlag, Heidelberg, 686-713, 2011.

World Bank: Dhaka: Improving Living Conditions for the Urban Poor, Bangladesh Development Series, Paper No. 17, The World Bank Office, Dhaka, 2007.

Zimmer, A.: Everyday governance of the waste waterscapes. A Foucauldian analysis in Delhi's informal settlements, Department of Geography, University of Bonn, Bonn, 2012.

Zimmer, A. und Sakdapolrak, P.: The Social Practices of Governing. Analysing Waste Water Governance in a Delhi Slum, Urbanization and Environment ASIA, 3, 325-341, 2012. 